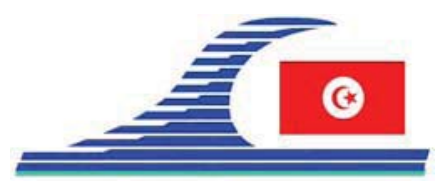

\author{
Conférence Méditerranéenne Côtière et Maritime \\ EDITION 1, HAMMAMET, TUNISIE (2009) \\ Coastal and Maritime Mediterranean Conference \\ Disponible en ligne - http://www.paralia.fr-Available online
}

\title{
Sédiments de dragage : variabilité des sédiments, retour d'expérience sur les pratiques de dragage et sur la valorisation à terre en France
}

\section{Lionel MANCIOPPI ${ }^{1}$, Anne BENARD ${ }^{1}$, Pierre HENNEBERT ${ }^{1}$, Benoit HAZEBROUCK ${ }^{2}$}

1. INERIS, Direction des Risques Chroniques, plate forme expérimentale ARDEVIE, Domaine du Petit Arbois, BP 33,13545 Aix en Provence, France.

Lionel.mancioppi@ineris.fr

2. INERIS, Direction des Risques Chroniques, Parc Technologique ALATA, $\mathrm{BP}^{\circ}{ }^{\circ}$ 2, 60550 Verneuil-en-Halatte, France.

\section{Résumé :}

L'accumulation de sédiments dans les ports, les chenaux d'accès, les canaux, les rivières et les fleuves empêche la circulation des bateaux et, lorsqu'ils sont pollués, impacte la qualité chimique des milieux aquatiques. Des opérations de dragage sont réalisées pour rétablir le bon usage ou la bonne qualité des eaux.

En France, les quantités annuelles de sédiments dragués pour l'entretien des cours d'eau, fleuves et canaux sont de l'ordre de 5 millions de $\mathrm{m}^{3}\left(\mathrm{Mm}^{3}\right)$ et de l'ordre de $50 \mathrm{Mm}^{3}$ pour les ports maritimes. La création de structures nouvelles génère également des quantités de sédiments de dragage non incluses dans ces chiffres.

Les techniques de remise en suspension et/ou d'immersion sont les plus couramment utilisées, mais elles sont désormais restreintes, au titre de la réglementation sur l'eau. Les quantités gérées à terre (dépôts, régalage, valorisation en techniques routières, aménagement et comblement de carrière...) sont estimées à environ $2 \mathrm{Mm}^{3} /$ an pour les sédiments d'eau douce et de l'ordre de $5 \mathrm{Mm}^{3}$ /an pour les sédiments marins.

Face à de tels volumes, l'enjeu est de permettre une gestion à terre sécurisée sur le plan environnemental et juridique. Le Grenelle de l'Environnement et la loi associée ont souligné la nécessité d'une amélioration de la gestion des sédiments.

La caractérisation de la variabilité des sédiments de dragage vis-à-vis des transferts de contaminants est essentielle pour permettre de définir des filières de gestion à terre viables pour les gros volumes concernés. L'INERIS réalise actuellement un recueil de données et une étude statistique sur la variabilité physico-chimique des sédiments marins et terrestres à l'échelle nationale française, en teneur brute et en relargage. Cette étude s'appuie sur les données de Voies Navigables de France (VNF), des Agences de l'Eau, du Réseau national de surveillance de la qualité de l'Eau et des sédiments des POrts Maritimes (REPOM) de l'IFREMER et du projet SEDIMARD 83, de 1994 à 2008.

DOI: $10.5150 / \mathrm{cmcm} .2009 .033-6$ 
Actuellement, l'étude rassemble 44000 données portant sur la granulométrie, la teneur en carbone organique, en métaux toxiques, en composants organiques, sur des caractérisations microbiologiques, ainsi que sur des tests d'écotoxicologie pour un ensemble de 2500 échantillons de sédiments fluviaux et marins. Le tableau ci-dessous présente les teneurs minimales et maximales rencontrées dans les données à ce jour traitées.

Cette étude s'est enrichie d'un retour d'expérience sur les pratiques des opérations de dragage et de valorisation à terre des sédiments au regard de l'impact potentiel sur le milieu aquatique. L'intervention de l'INERIS présentera les conclusions préliminaires de cette étude, qui s'achèvera fin décembre 2009.

Tableau 1. Teneurs minimales et maximales des composés organiques et des métaux présents dans les sédiments fluviaux et marins, issues des données à ce jour traitées.MS: matière sèche, HAP : hydrocarbures aromatiques polycycliques, PCB : polychlorobiphényles.

\begin{tabular}{lllll}
\hline Sédiments & \multicolumn{2}{l}{ FLUVIAUX } & \multicolumn{2}{l}{ MARINS } \\
\hline Composés Organiques $(\boldsymbol{\mu g} / \mathbf{k g}$ MS) & min & max & min & max \\
\hline HAP Totaux & 0.2 & 3363 & 0.78 & 231750 \\
Hydrocarbures totaux & & & 1000 & 8900000 \\
Monobutylétain & & & 0.01 & 1198 \\
PCB totaux & 100 & 1200 & 0.08 & 1810 \\
Tributylétain & & & 0.005 & 152000 \\
Dibutylétain & & & 0.07 & 3600 \\
Triphénylétain & & & 10 & 59 \\
Métaux (mg/kg MS) & & & & \\
\hline Arsenic & 0.1 & 368 & 0.094 & 68.5 \\
Cadmium & 0.2 & 2226 & 0.03 & 8.6 \\
Chrome & 2.0 & 1922 & 1 & 636 \\
Cuivre & 2.0 & 1842 & 2 & 2651 \\
Mercure & 0.1 & 88 & 0.01 & 50 \\
Nickel & 4.0 & 625 & 1 & 214 \\
Plomb & 2.0 & 16670 & 5 & 662 \\
Zinc & 31.9 & 17663 & 1.54 & 2800 \\
\hline
\end{tabular}

\section{Références}

ALZIEU C. (1991). Environmental problems caused by TBT in France: assessment, regulation and prospects. Marine Environmental Research, 32, pp 7-17.

BENARD A. (2009). Rapport d'étude, «Impacts sur les milieux aquatiques des sédiments de dragage gérés à terre: Problématique, contexte réglementaire, modélisation du transfert de contaminants organiques». DRC-08-95306-16457A, 64 p. 